\title{
Towards a Novel Safety Norm for Domestic Robotics
}

\author{
Martin Wassink and Stefano Stramigioli \\ Control Engineering, EE-Math-CS, University of Twente \\ P.O. Box 217, 7500 AE, Enschede, The Netherlands \\ m.wassink@utwente.nl_s.stramigioli@utwente.nl
}

\begin{abstract}
Safety is a critical success factor for consumer acceptance of domestic robotic products. Some researchers have adopted the Head Injury Criterion (HIC) as absolute safety norm. However, this norm covers only part of the safety risk. In many cases skin damage (e.g. cuts, wounds, etc) can be a more serious risk.

This article shows how to work towards a novel absolute safety measure for evaluating the shape and material choices of a robotic design w.r.t. skin damage. The proposed safety norm evaluates the situation of an unintended uncontrolled collision of a robotic part against a human. Maximum curvatures of the exterior robotic shape are approximated as a sphere in contact with the human skin (locally approximated as a flat surface). This local spheric approximation of the impact contact is used to predict maximum tensile stress during impact of the robotic part on the human. Robotic designs that include points for which the tensile strength of the skin is exceeded will cause at least skin fracture and are therefore considered intrinsically unsafe.
\end{abstract}

While in general applicable, this paper specifically addresses how to apply the proposed norm in the case of safety evaluation of robotic manipulators.

\section{INTRODUCTION}

Domestic robotics is a fast emerging commercial application domain. For several decades robotic technologies have been fruitfully used in industry to automate production processes. Today, kids can play with robotic toys and only recently robotic vacuum cleaners and lawn mowers became commercially available. These are examples of the emerging service and personal robotics market.

Domestic robotics is evolving towards full scale service robots, equipped with sophisticated soft- and hardware for personal assistance, entertainment, rehabilitation etc. The experimental service robot of DLR, Robutler [1], is one of the nice examples currently demonstrated. These service robots execute their tasks in the unstructured human environment, posing thorough safety requirements on the design of service robots.

The need for safety in robotics was already identified by Asimov in 1942 [2]. Nowadays, for industrial robotics, standards define requirements on the robotic device and its working environment, e.g. ISO 10218-1 (2006) [3]. As domestic robotics emerges, robotic safety has become an important item on the research agenda. It is up to the robotics society to evaluate their robotic products on safety. Nevertheless, safety legislation for domestic robotic products is far behind on technological advances.
In this paper, the authors address the need for absolute safety measures in domestic robotics. Furthermore, as a first step towards a novel safety measure, a novel absolute safety measure is formalized to evaluate the safety of a robotic design based on its cover shape and material. The proposed safety measure is generally applicable to evaluate the safety (w.r.t. skin damage) of any part of a robotic device. In this paper, the authors specifically address the application of safety evaluation of robotic manipulators.

Section II will first elaborate somewhat more on safety considerations in robotic design, Section III gives a brief overview on related work, Section IV presents the proposed novel safety measure, followed by some conclusions in Section V. Finally, Section VI discusses projected future work.

\section{SAFETY CONSIDERATIONS}

As opposed to industrial applications, in domestic robotics, the design of the operational environment can not be influenced and the need for intrinsically safe robotic designs rises. For hazards (e.g. breakage) on lifeless objects, certain risk levels ('probability' $\times$ 'severity') could be accepted. However, for human beings, lethal severity is unacceptable. Therefore, intrinsically safe design refers to a design which can not ("probability' $=0$ ) cause unacceptable harm to human beings.

For the robotic safety considerations, the authors focus on mechanical hazards to humans, for which three types of possible harmful injuries are distinguished :

- Static pressure injuries: strangle, squeezing;

- Shock injuries: internal bleeding, bone fracture, brain damage [4];

- Skin injuries: bruise, cut, laceration.

During (quasi) static human-robot interaction, static pressure applied to the human body can cause e.g. squeezing or strangle. Unintended collisions are found to be a worst case scenario of dynamic human-robot interaction, causing either shock or skin injuries.

In any of these situations, independent of the control, an intrinsically safe robot should not be able to cause any of these injuries with unacceptable severity. Quantitative absolute norms (as apposed to a relative norm) allow us to put a number on 'unacceptable severity', representing e.g. lethal or painful impact, whichever is decided upon. 
Whenever software fails (delayed reaction time, sensor failure, decision fault, wrong velocity limitation, etc), the mechanical hardware of the robot should be such that intrinsic safety is still ensured. Thus, intrinsic safety is determined by the mechanical robotic design, whereas the softwarehardware combination should determine functionality and probability of occurrence of acceptable severities.

In the case of an uncontrolled (or erroneously controlled) situation, conventional industrial robot arms (stiff and high inertias) will cause high impact loads during human robotcollision, resulting in e.g. possible lethal shock injuries. Therefore, these arms are not intrinsically safe, making conventional arms (apart from other practical reasons) not applicable for domestic robotics.

In domestic robotics, other mechanical concepts are needed for the manipulators, involving different design tradeoffs to maintain intrinsic safety and functional performance. Several conceptual design novelties are introduced in e.g. [1], [5] and [6]. These robotic arms are designed to reduce impact loads in the case of unintended collisions. Although it is recognized that the mechanisms can indeed reduce impact loads, many solutions still depend on software decisions (e.g. variable stiffness). It remains debatable whether these concepts are intrinsically safe. Therefore, measurable absolute safety norms are needed for each of the three types of injury risks to quantify the intrinsic safety of robotic designs.

\section{RELATED WORK}

Some effort has been put forward in the field of robotic safety assessment. In medical robotics qualitative certification proposals (e.g. [7]) can be found, being safety procedures which are meant to ensure that engineers have performed a successful risk analysis. However, this does not ensure design for intrinsic safety.

Furthermore, in [4] an overview on possible safety strategies and a relative safety evaluation method is reported. This method is not yet fully worked out as an absolute safety norm, nor a calculation method was found validated by the authors.

The following sections reveal some of the current state of research on absolute safety norms in robotics.

\section{A. Static Pressure Injuries}

For this static situation it is likely that as long as a certain human can apply more force to the robot than vice versa, then this human can save himself from a severe static humanrobot interaction situation. Clearly, using this idea to design a robot which can not generate more force than any human will be difficult, since all human beings have different strengths, builds, etc. Clever mechanical hardware solutions are needed to guarantee intrinsic safety w.r.t. static pressure injuries, while remaining task capability. No research on safety norms or mechanical solutions for this topic were found.

\section{B. Shock Injuries}

For quantifying acceleration (shock) traumas to the head, both [8], [6] and [5] proposed the Head Injury Criterion
(HIC) as quantitative severity measure for unintended blunt impacts to the head. The HIC discriminates between severe and less severe traumatic brain injuries caused by internal stretching and tearing of (brain) tissue [9]. The HIC is borrowed from automotive and sports industry, where crash tests on dummies have been common practice for a long time. The HIC is defined as [10]:

$$
H I C=\max _{t_{1}}\left\{\left(\frac{1}{\Delta} \int_{t_{1}}^{t_{1}+\Delta} a(\tau) d \tau\right)^{2.5} \cdot \Delta\right\}
$$

where $a(\tau)$ is the head acceleration measured in $g$ as function of time and $t_{1}+\Delta$ is a time interval within the measurement interval starting at $\tau=t_{1}$, chosen such that the integral is maximized (i.e. worst velocity change during impact). Life critical values for the $H I C_{15}$ range from 390 for small children to 700 for adults [10], where subscript 15 refers to $\Delta=15 \mathrm{~ms}$.

Improvements on the use of the HIC are still going on, see e.g. [10]. Also criticism is found on the use of the HIC. For example, the HIC allows for short unsafe acceleration pulses [11]. The HIC does not discriminate between different types of injuries [9]. Researchers are looking for next generation head injury indices based on head models [9]. Assuming the head is the most vulnerable part of the human, the HIC could be used to set a worst case value for acceleration severities of the human body. Blunt impact mechanisms on other parts of the human body are discussed in [12].

\section{Skin Injuries}

Depending on robotic shape, cover material and velocity, an unintended human-robot collision might not cause too much shock injury, but can still cause unacceptable severe skin injuries. This is partially mentioned in [4]. However, the safety evaluation method reported by those authors, gives a relative measure and lacks the incorporation of a contact model, material properties and robot speed.

In this paper a novel absolute safety measure is introduced that will allow for quantification of the severity of skin injuries, caused by unintended human-robot collisions.

\section{NOVEl SAFETy MEASURE Skin INJURIES}

\section{A. Injury Analysis}

Depending on the impact place on the human body and the robot properties (cover material, velocity and shape), different skin injuries can be caused during impact, such as:

- Bruise: skin discoloration and pain, caused by pressure on skin leading to breakage of capillaries in skin;

- Laceration: irregular cuts, caused by a blunt impact to soft tissue which lies over hard tissue;

- Cut: a break or opening in the skin, caused by clean sharp edge.

Also all kinds of skin penetrations can happen. It is assumed that in the mechanical process of penetrating the skin, first one of the above mentioned injury states are passed. These identified injuries are caused by exceeding certain stress levels of the skin, during impact. Lower stresses may cause 


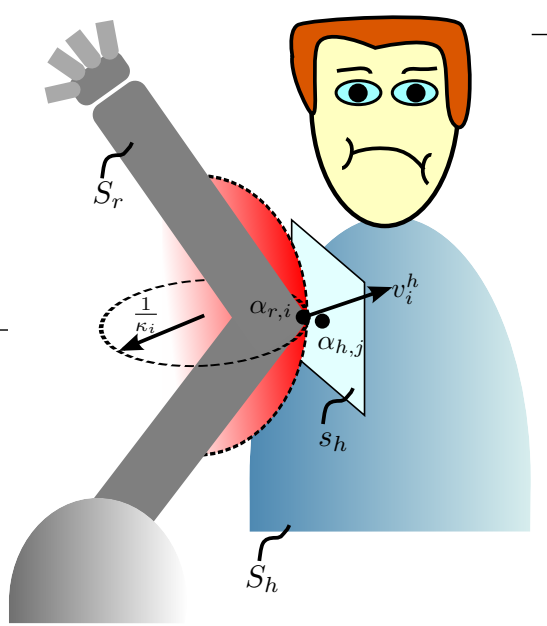

Fig. 1. Robot arm part $\alpha_{r, i} \in S_{r}$ with linear velocity $v_{i}^{h}$ hits human body part $\alpha_{h, j} \in s_{h}$, where contact surface on human is approximated by local flat surface $s_{h} \subseteq S_{h}$ perpendicular to translational impact velocity. Local arm shape is determined by largest curvature $\kappa_{i}$ through $\alpha_{h, j}$.

bruises, whereas exceeding the tensile strength of skin causes skin breakage (cuts or lacerations).

The difference in cuts and lacerations is found in the fact that for cuts, skin stresses are build up on one place (or line), whereas in lacerations multiple stress regions are found, causing an irregular wound. Therefore, cuts can only be caused by sharp edges, which are obviously unsafe, while bruises, or worse, lacerations, are caused by blunt edges. It is therefore obviously stated that sharp edges are unsafe.

Therefore the proposed safety measure quantifies the severity of combinations of shape bluntness, cover material and velocity.

\section{B. Impact Formalization}

As previously analyzed, maximum stress levels during impact on the human skin are a severity measure for the impact. The proposed safety norm evaluates the maximum stress that will occur during impact of a point on the robotic cover against a human body part. First the human-robot impact is formalized.

Fig. 1 shows the impact under consideration. Consider the point $\alpha_{r, i}$ on the robot arm cover surface $S_{r}$ and the point $\alpha_{h, j}$ on the human body surface $S_{h}$. For simplicity, both points are considered to have homogeneous material properties, i.e. Young's modulus $E_{r, i}$ and $E_{h, j}$ and Poisson ratios $\nu_{r, i}$ and $\nu_{h, j}$. The shape of the robot arm in $\alpha_{r, i}$ is defined by the worst case curvature through $\alpha_{r, i}$, determined by the largest principal curvature $\left(\kappa_{i}\right)$ through $\alpha_{r, i}$. The surrounding surface of the human body point $\alpha_{h, j}$ is approximated as a flat half-space surface $s_{h} \subseteq S_{h}$. The translational impact of point $\alpha_{r, i}$ is examined along a line, perpendicular to the local surface $s_{h}$.

On impact, the robot arm has a certain configuration $q \in$ $Q^{n}$, where $Q^{n}$ represents the complete configuration space of the $n$-DoF robotic arm, and the complete arm has kinetic

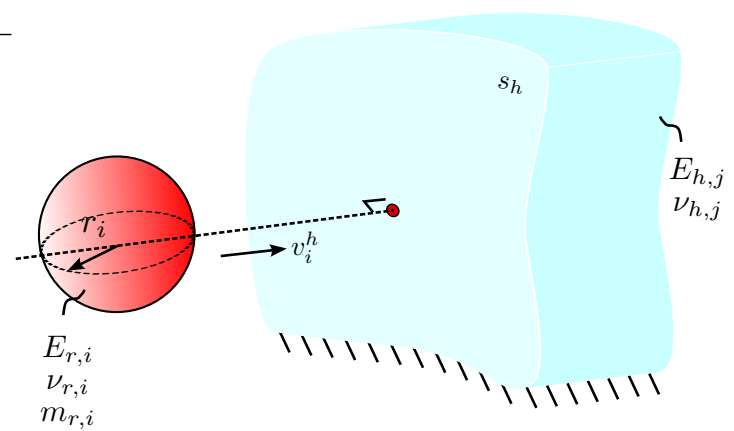

Fig. 2. Impact model of robot point $\alpha_{r, i} \in S_{r}$ hitting human body part $\alpha_{h, j} \in S_{h}$.

co-energy $U_{k}^{*}$ :

$$
U_{k}^{*}(q, \dot{q})=\frac{1}{2} \dot{q}^{T} M(q) \dot{q}
$$

where $M(q)$ is the configuration dependent inertia matrix of the whole manipulator.

For the point $\alpha_{r, i}$, an effective linear translational velocity $v_{i}^{h}$ is defined together with a configuration dependent effective mass $m_{r, i}(q)$ along the line of impact. Superscript $h$ in the linear velocity indicates that the velocity is expressed w.r.t. the human body contact surface coordinates $\left(s_{h}\right)$. Both $v_{i}^{h}$ and $m_{r, i}(q)$ are scalar quantities, since they are defined along the normal direction of the impact surface. Together they determine the effective kinetic co-energy of $\alpha_{r, i}, \Delta U_{k, i}^{*}$, on impact:

$$
\Delta U_{k, i}^{*}=\frac{1}{2} m_{r, i}(q)\left(v_{i}^{h}\right)^{2}
$$

Since safety considerations are examined, the worst case situation is considered, in which the human is fixed to his position and no energy is lost due to friction or damping. For these assumptions it holds that maximum impact stress is reached when all effective kinetic energy of $\alpha_{r, i}$ is transferred into potential energy $V_{d}(\delta)$, stored in elastic surface deformation $(\delta)$.

Thus, the effective kinetic energy $\Delta U_{k, i}$ is that part of the total kinetic energy of the manipulator that is transferred into $V_{d}(\delta)$ during impact. As a result of multiple degrees of freedom (not all joints have to lose their kinetic energy during impact) and possible manipulator compliance, the effective kinetic energy does not have to be equal to the total kinetic energy of the manipulator:

$$
\Delta U_{k, i} \leq U_{k}(q, \dot{q})
$$

The configuration and possible compliant mass decoupling determine the effective impact mass $m_{r, i}(q)$ to account for in the effective kinetic energy of the modeled impact of $\alpha_{r, i}$.

The worst case shape approximation results in modeling the impact of $\alpha_{r, i}$ on $\alpha_{h, j}$, as the impact of a sphere with radius $r_{i}=\frac{1}{\kappa_{i}}$, material properties $\left(E_{r, i}, \nu_{r, i}\right)$ and effective kinetic energy $\Delta U_{k, i}$ on a flat fixed half-space surface with material properties $\left(E_{h, j}, \nu_{h, j}\right)$. The impact situation under consideration is reduced to a parameterized impact model as shown in Fig. 2. 


\section{Effective Kinetic Energy for Rigid Manipulator}

For a rigid manipulator, the effective kinetic energy that is transferred into potential energy during impact due the loss of momentum in the perpendicular impact direction (i.e. $v_{i}^{h}=0$ at maximum impact) is derived by Duindam \& Stramigioli [13]:

$$
\begin{aligned}
\Delta U_{k, i}^{*} & =\frac{1}{2} \dot{q}^{T}\left(t_{-}\right)\left(A\left(A^{T} M(q)^{-1} A\right)^{-1} A^{T}\right) \dot{q}\left(t_{-}\right) \\
& =\frac{1}{2} \dot{q}^{T}\left(t_{-}\right) P \dot{q}\left(t_{-}\right)
\end{aligned}
$$

where $P:=A\left(A^{T} M(q)^{-1} A\right)^{-1} A^{T}, t_{-}$indicates the time instant just before impact and the transpose of the $n$-DoF vector $A(q)$ is used to compute the linear velocity $v_{i}^{h}(t)=$ $A^{T}(q) \dot{q}(t)$ of the point $\alpha_{r, i}$ w.r.t. the human body contact surface coordinates $\left(s_{h}\right)$.

Substituting the linear velocity $v_{i}^{h}=A^{T}(q) \dot{q}$ into Eq. 3 and equating to Eq. 5 results in the following equality:

$$
\Delta U_{k, i}^{*}=\frac{1}{2} m_{r, i} \dot{q}^{T}\left(t_{-}\right) A A^{T} \dot{q}\left(t_{-}\right)=\frac{1}{2} \dot{q}^{T}\left(t_{-}\right) P \dot{q}\left(t_{-}\right)
$$

Rearranging gives:

$$
\dot{q}^{T}\left(t_{-}\right)\left(P-m_{r, i} A A^{T}\right) \dot{q}\left(t_{-}\right)=0 \quad \forall \dot{q}(t)
$$

giving the sufficient condition:

$$
P-m_{r, i} A A^{T}=0
$$

such that an analytical expression for $m_{r, i}$ is found:

$$
m_{r, i}(q)=\left(A(q)^{T} M^{-1}(q) A(q)\right)^{-1}
$$

\section{General Skin Injury Severity Measure}

To determine the maximum impact stress $\left(\sigma_{i, j, q}\right)$ during impact of robot cover point $\alpha_{r, i} \in S_{r}$ in configuration $q \in Q^{n}$ against human body point $\alpha_{h, j} \in S_{h}$, a contact model is needed that determines the potential energy of the surface deformation $V_{d, j}$ as a function of material properties, material structure and the total shape deformation $(\delta)$ along the impact line, i.e. the displacement of mutual approach of distant points in the robotic arm part and the human body part:

$$
V_{d, j}=f_{1}\left(\delta, E_{r, i}, \nu_{r, i}, r_{i}, E_{h, j}, \nu_{h, j}\right)
$$

Subscript ${ }_{j}$ in $V_{d, j}$ refers to the potential energy function on human body part $\alpha_{h, j}$, implying that the potential energy function is not only a function of its variables, but the function itself may also vary due to different human body structures on different human body parts $\alpha_{h, j}$. The potential energy is equated to the effective kinetic energy $V_{d, j}=\tilde{U}_{k, i}$, such that rewriting the equations yields a function for the maximum shape deformation $\delta_{\max , j}$ :

$$
\delta_{\max , j}=f_{2}\left(E_{r, i}, \nu_{r, i}, r_{i}, E_{h, j}, \nu_{h, j}, m_{r, i}, v_{i}^{h}\right)
$$

The maximum impact stress on the human body point for the impact under consideration, will be a function of the maximum shape deformation, s.t.

$$
\sigma_{i, j, q}=f_{3}\left(E_{r, i}, \nu_{r, i}, r_{i}, E_{h, j}, \nu_{h, j}, m_{r, i}, v_{i}^{h}\right)
$$

which is the desired quantified severity measure, proposed in this article.

Evaluating the total robot arm design on all possible configurations and impact points against the complete human body for maximum possible velocity $v_{i}^{h}$ (i.e. function of $\dot{q}$ ) gives us the worst case severity $\sigma_{\max }$ of a robotic arm design:

$$
\sigma_{\max }=\max \left(\sigma_{i, j, q}(\cdot) \mid q, \dot{q} \in T Q^{n}, \alpha_{r, i} \in S_{r}, \alpha_{h, j} \in S_{h}\right)
$$

A safety statement should incorporate a maximum allowable stress level $\left(\sigma_{h}\right)$ on the human body, such that a robotic design is safe w.r.t. skin injuries if:

$$
\sigma_{\max } \leq \sigma_{h}
$$

and unsafe otherwise.

However, evaluating all possible combinations is of course practically undesirable. Therefore the authors point out that for bruises as well as lacerations, the human body areas where thin soft tissue layers lie over hard tissue material are the most critical human body parts with regard to easily building up high impact stresses. These areas are found on the head, around joints, the chest, etc. Furthermore one worst case effective mass $m_{r}$ is assumed for the robotic surface subset $s_{r} \subseteq S_{r}$ under consideration, defined as:

$$
m_{r}=\max \left(m_{r, i}(q) \mid q, \dot{q} \in T Q^{n}, \alpha_{r, i} \in s_{r}\right)
$$

With these assumptions, the number of variables is reduced such that the severity of a point $\alpha_{r, i} \in s_{r}$ on the robotic arm subsurface $s_{r}$ can be evaluated by one calculation, depending only on robotic arm parameters:

$$
\sigma_{i}=f_{4}\left(E_{r, i}, \nu_{r, i}, r_{i}, v_{i}^{h}\right)
$$

Taking the intrinsic material properties of the robotic arm point $\alpha_{r, i}$ together as one material constant $\Gamma_{i}$ :

$$
\Gamma_{i}=f_{5}\left(E_{r, i}, \nu_{r, i}\right)
$$

gives a comprehensive quantitative worst case severity measure for the evaluation of robotic cover points $\alpha_{r, i} \in s_{r}$ of a robotic design with a worst case mass $m_{r}$ :

$$
\sigma_{i}=f_{6}\left(\Gamma_{i}, r_{i}, v_{i}^{h}\right)
$$

By equating $\sigma_{i}\left(\Gamma_{i}, r_{i}, v_{i}^{h}\right)=\sigma_{h}$, a 3D boundary surface $\Gamma_{i}\left(r_{i}, v_{i}^{h}\right)$ is found, separating safe and unsafe combinations of material properties, shape and velocity for $\alpha_{r, i} \in s_{r}$. This boundary surface provides a quick look up to verify whether a point on the robot design complies to the safety region.

\section{E. First Proposal Contact Model}

For the general severity skin injury measure to be usable, a contact model is needed for the worst case situation of spheric impact on a double layered flat half-space. Fig. 3 shows this worst case contact with a thin (ca. $3 \mathrm{~mm}$ ) skin layer with material properties $E_{s}$ and $\nu_{s}$ and a fixed thick layer of bone, $E_{b}$ and $\nu_{b}$.

From literature, see e.g. Yamada [14], it is known that skin has limited tensile strength: $\sigma_{h}=1 e 6 \mathrm{~N} / \mathrm{m}^{2}$. As a first proposal to determine an approximate function for worst 


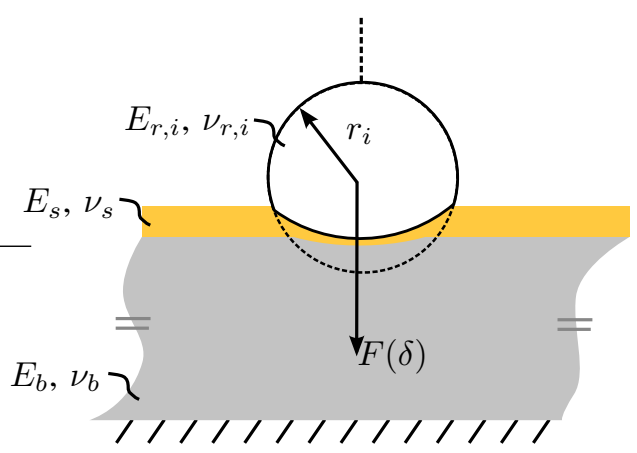

Fig. 3. Spheric contact model for worst case double layer contact; thin soft skin $\left(E_{s}, \nu_{s}\right)$ over thick fixed bone $\left(E_{b}, \nu_{b}\right)$.

case tensile impact stresses between sphere, skin and bone, it is assumed that the contact behavior is predominantly determined by the material properties of the human bone and the sphere (i.e. approximated robot contact point $i$, with properties $\left.E_{r, i}, \nu_{r, i}\right)$. Therefore, quasi-static Hertzian contact is modeled between sphere and bone. Furthermore, the incompressible skin layer in between, is assumed to take over the contact pressure and (by incompressible deformation) equally distributes the contact pressure over the complete contact surface between sphere and bone. Outside the contact area, no contact pressure exists in the skin. The largest pressure gradient in the skin arises at the contact edges, which induces tensile stress at the edges of the contact, being the proposed severity measure.

Under the assumption of homogeneous and isotropic frictionless contacting bodies, small (in relation to $r_{i}$ ) contact area, elastic and localized deformations, the tensile stress function, $\sigma_{i}\left(\Gamma_{i}, r_{i}, v_{i}^{h}\right)$, is deduced from Hertzian contact theory ([15]) as follows.

The total shape deformation along the impact line requires force $F(\delta)$, given by [15]:

$$
F(\delta)=\delta^{\frac{3}{2}} \cdot\left(\frac{4 E_{c}}{3}\right) \cdot r_{i}^{\frac{1}{2}}
$$

where $E_{c}$ is the effective contact modulus (assuming spherebone contact):

$$
\frac{1}{E_{c}}=\frac{1-\nu_{r, i}^{2}}{E_{r, i}}+\frac{1-\nu_{b}^{2}}{E_{b}}
$$

Integrating $F(\delta)$ over $\delta$, yields the potential energy function:

$$
V_{d}\left(\delta, E_{c}, r_{i}\right)=\int_{\delta} F(\delta) \cdot d \delta=\frac{2}{5} \delta^{\frac{5}{2}} \cdot\left(\frac{4 E_{c}}{3}\right) \cdot r_{i}^{\frac{1}{2}}
$$

such that after equating to Eq. 3 and assuming worst case effective mass $m_{r}, \delta_{\max }$ is found:

$$
\delta_{\max }=\left(\frac{15 \cdot m_{r}}{16 \cdot E_{c} \cdot r_{i}^{\frac{1}{2}}}\right)^{\frac{2}{5}} \cdot v_{i}^{\frac{4}{5}}
$$

Then $\delta_{\max }$ determines the maximum force $F\left(\delta_{\max }\right)$ during impact, which induces maximum contact pressure $p_{0}$ :

$$
p_{0}=\frac{1}{\pi}\left(\frac{6 F\left(\delta_{\max }\right) E_{c}^{2}}{r_{i}^{2}}\right)^{\frac{1}{3}}
$$

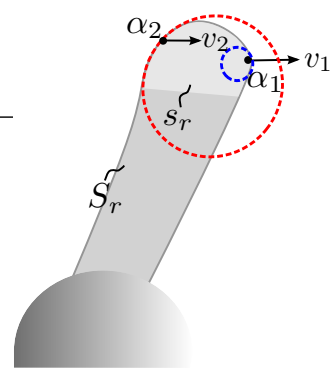

Fig. 4. Simple 1-DoF robot arm example. Points in $s_{r} \subset S_{r}$ are under consideration with effective worst case mass $m_{r}=4.5 \mathrm{~kg}$. Points $\left(\alpha_{1}, \alpha_{2}\right) \in s_{r}$ are numerically evaluated, $r_{1}=0.01 \mathrm{~m}$ and $r_{2}=0.03 \mathrm{~m}$.

The maximum tensile stress for spheric contact and maximum contact pressure $p_{0}$ in material $x$, is given by:

$$
\sigma_{x}=\frac{p_{0}\left(1-2 \nu_{x}\right)}{3}
$$

which results in the following expression for the maximum tensile stress in the skin during the impact under consideration:

$$
\sigma_{i}\left(r_{i}, E_{c}, v_{i}\right)=\frac{p_{0}\left(1-2 \nu_{s}\right)}{3}=\beta \frac{E_{c}^{\frac{4}{5}} \cdot m_{r}^{\frac{1}{5}} \cdot v_{i}^{\frac{2}{5}}}{r_{i}^{\frac{3}{5}}}
$$

with

$$
\beta=\frac{\left(1-2 \nu_{s}\right) \cdot 6^{\frac{1}{3}} \cdot\left(\frac{4}{3}\right)^{\frac{1}{3}} \cdot\left(\frac{15}{16}\right)^{\frac{1}{5}}}{3 \pi}
$$

Finally, by introducing the robotic arm material function:

$$
\Gamma_{i}=\frac{E_{r, i}}{1-\nu_{r, i}^{2}}
$$

and equating $\sigma_{i}\left(r_{i}, E_{c}, v_{i}\right)=\sigma_{h}$, the maximum tensile stress function is rewritten into the $3 \mathrm{D}$ safety boundary surface function:

$$
\Gamma_{i}\left(r_{i}, v_{i}\right)=\left(\left(\frac{\beta \cdot m_{r}^{\frac{1}{5}} \cdot v_{i}^{\frac{2}{5}}}{\sigma_{h} \cdot r_{i}^{\frac{3}{5}}}\right)^{\frac{5}{4}}+\frac{\nu_{b}^{2}-1}{E_{b}}\right)^{-1}
$$

for fixed $\sigma_{h}$ and worst case effective mass $m_{r}$.

\section{F. Numeric Example}

As a simple example, a 1-DoF robot arm is considered, as shown in Figure 4. The safety of points in $s_{r} \subset S_{r}$ are examined, with worst case effective mass $m_{r}=4.5 \mathrm{~kg}$, $E_{b}=17 \mathrm{~N} / \mathrm{m}^{2}, \nu_{b}=0.3, \nu_{s}=0.45$ and $\sigma_{h}=12 \mathrm{~N} / \mathrm{m}^{2}$ (numbers taken from [14], [16] and [17]). With Eq.19 the according safety boundary surface is plotted, presented in Fig. 5.

Two points are numerically evaluated: $\alpha_{1}$ has material properties of ABS plastic $\left(\Gamma_{1}=3 e 9 \mathrm{~N} / \mathrm{m}^{2}\right)$ and its shape is defined by $r_{1}=0.01 \mathrm{~m}, \alpha_{2}$ has oak wood material $\left(\Gamma_{1}=\right.$ $13 e 9 \mathrm{~N} / \mathrm{m}^{2}$ ) with $r_{2}=0.03 \mathrm{~m}$. Fig. 5 clearly shows that point $\alpha_{1}$ is safe as long as its effective velocity $\left(v_{1}\right)$ is smaller than $1 \mathrm{~m} / \mathrm{s}$; however, for point $\alpha_{2}$ the velocity must not exceed $0.1 \mathrm{~m} / \mathrm{s}$. Either softer materials or smoother edges should be considered for $\alpha_{2}$ to allow for larger velocities. 


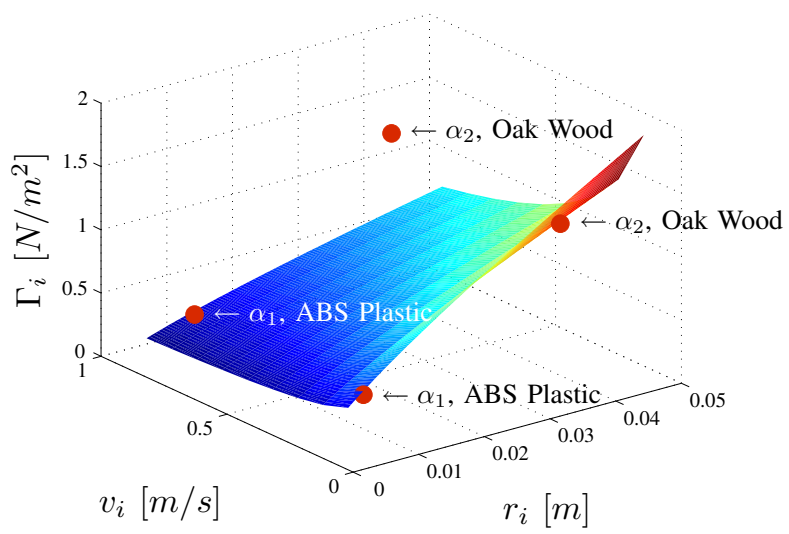

Fig. 5. 3D safety boundary surface $\Gamma_{i}\left(r_{i}, v_{i}\right)$ for robot sub-surface $s_{r}$ with effective mass $m_{r}=4.5 \mathrm{~kg}$, using the interpreted Hertzian contact model. All combinations of $\left(\Gamma_{i}, r_{i}, v_{i}\right)$ under the surface are considered safe.

Otherwise, in order to keep this robot arm intrinsically safe, it must be ensured that the mechanical hardware is not able to produce velocities larger than $0.1 \mathrm{~m} / \mathrm{s}$.

Furthermore, Fig. 5 easily shows the design trade-offs for the robotic surface area under consideration $\left(s_{r}\right)$.

\section{COnClusions}

In this paper the need for intrinsically safe mechanical design of e.g. robotic arms for domestic robotics has been discussed. In this emerging field, safety will be a critical success factor. In order to quantify safety, absolute safety measures are necessary. It is pointed out that only one safety measure was found in literature, i.e. HIC. The HIC evaluates the survival probability of head shaking, originally used in car industry. However head shaking is not the only hazard in human-robot interaction.

A general framework is set up to work towards a novel absolute safety measure, quantifying skin damage injuries as a function of robotic design parameters. The severity of a certain robotic design is evaluated on its material properties, shape and allowable velocity. Worst case unintended collisions are used to evaluate the design.

As a first proposal, the novel safety norm is quantified by modeling the impact contact, using a Hertzian contact model. It is shown that based on this model, a safety surface was generated that easily points out safe and unsafe design combinations. Therefore, the proposed safety measure can be used to find safe design trade offs during robot design.

\section{FUTURE WORK}

Future work will address further research on the layered (thin skin over bone) contact model and its validation by measurements and tests. Based on test data, human body parameter estimation values should be optimized, to work towards a reliable quantitative safety measure. furthermore, it would be promising to collaborate with medical and forensic researchers to gain further insights on injury mechanisms.

Furthermore, non perpendicular impacts with friction will also be important to investigate, since impact friction is another important mechanism that can induce skin stresses during impact.

In addition to the contact model, determining the effective mass for a compliant manipulator may not be trivial, and in general depends on multiple DoFs and configuration dependent compliances. Compliance can be of great help to increase the intrinsic safety by decoupling inertia. These considerations will also be accounted for in future research.

Other research projects should also be started to develop an intrinsically safe mechanical design that prevents static pressure injuries for all humans. A quantitative norm may be required.

\section{REFERENCES}

[1] U. Hillenbrand, B. Brunner, C. Borst, and G. Hirzinger, "The robutler: a vision-controlled hand-arm system for manipulating bottles and glasses," in CD, ISR 2004 35th International Symposium on Robotics, Paris-Nord Villepinte Exhibition Centre (France), 23-26 March 2004.

[2] I. Asimov, "Runaround," Astounding Science Fiction, March 1942.

[3] ISO 10218-1:2006 Robots for industrial environments - Safety requirements - Part 1: Robot, 2006.

[4] K. Ikuta, H. Ishii, and M. Nokata, "Safety evaluation method of design and control for human-care robots," Int. Journal of Robotics Research, vol. 33 , no. 5 , pp. $381-297,2003$.

[5] A. Bicchi and G. Tonietti, "Dealing with the safety-performance tradeoff in robot arms design and control: Fast and soft-arm tactics," IEEE Robotics and Automation Magazine, June 2004.

[6] M. Zinn, O. Khatib, B. Roth, and J. K. Salisbury, "A new actuation concept for human-friendly robot design: Playing it safe," IEEE Robotics and Automation Magazine, June 2004.

[7] J. Guiochet and A. vilchis, "Safety analysis of a medical robot for tele-echography," 2nd IARP IEEE/RAS joint workshop on Technical Challenge for Dependable Robots in Human Environments, pp. 217-227, 2002. [Online]. Available: http://www.laas.fr/g̃uiochet/\#Publications

[8] S. Oberer, M. Malosio, and R. D. Schraft, "Investigation of robothuman impact," in ISR 2006 - ROBOTIK 2006: Proceedings of the Joint Conference on Robotics, May 2006.

[9] D. Brands, "Predicting brain mechanics during closed head impact, numerical and costitutive aspects," Ph.D. dissertation, University of Eindhoven, Eindhoven, The Netherlands, 2002.

[10] R. Eppinger, E. Sun, F. Bandak, M. Haffner, N. Khaewpong, M. Maltese, S. Kuppa, T. Nguyen, E. Takhounts, R. Tannous, A. Zhang, and R. Saul, "Development of improved injury criteria for the assessment of advanced automotive restraint systems ii," National Highway Traffic Safety Administration (NHTSA) and National Transportation Biomechanics Research Center (NTBRC), Tech. Rep., 1999. [Online]. Available: http://www-nrd.nhtsa.dot.gov/

[11] J. Newman, "Criteria for head injury and helmet standards," NBEC Inc., Tech. Rep., 2005.

[12] D. J. Schenck and J. D. Bronzino, Biomechanics : Principles and Applications. CRC Press, 2003.

[13] V. Duindam and S. Stramigioli, "Optimization for mass and stiffness distribution for efficient bipedal walking," in Proceedings of the International Symposium on Nonlinear Theory and Its Applications, 2005.

[14] H. Yamada, Strength of Biological Materials, F. G. Evans, Ed. The Williams \& Wilkins Company, 1970.

[15] K. Johnson, Contact Mechanics. Cambridge University Press, 1985.

[16] Y. C. Fung, Biomechanics: Mechanical Properties of Living Tissues. Springer, 2005

[17] S. Diridollou, V. Vabre, M. Berson, L. Vaillant, D. Black, J. M. Lagarde, J. M. Grgoire, Y. Gall, and F. Patat, "Skin ageing: changes of physical properties of human skin in vivo," International Journal of Cosmetic Science, vol. 23, no. 6, p. 353, 2001. 